\title{
Context-based analysis of urban air quality using an opportunistic mobile sensor network
}

\author{
Xuening Qin ${ }^{1,3(\varangle)}$, Ljiljana Platisa ${ }^{1,3}$, Tien Huu Do ${ }^{2,3}$, Evaggelia \\ Tsiligianni $^{2,3}$, Jelle Hofman ${ }^{4}$, Valerio Panzica La Manna ${ }^{4}$, Nikos Deligiannis ${ }^{2,3}$, \\ and Wilfried Philips ${ }^{1,3}$ \\ 1 imec-TELIN-IPI, Department of Telecommunications and Information Processing, \\ Ghent University, Sint-Pietersnieuwstraat 25, B-9000 Ghent, Belgium \\ 2 imec-ETRO, Department of Electronics and Informatics, Vrije Universiteit Brussel, \\ Pleinlaan 2, B-1050 Brussels, Belgium \\ 3 imec, Kapeldreef 75, B-3001 Leuven, Belgium \\ 4 Holst Center, imec, High Tech Campus 31, 5656 AE Eindhoven, The Netherlands \\ Xuening.Qin@ugent.be
}

\begin{abstract}
Air pollution is becoming an important environmental issue and attracting increasing public attention. In urban environments, air pollution changes very dynamically both with time and space and is affected by a large variety of factors such as road type, urban architecture, land use and variety of emission sources. In order to better understand the complexity of urban air pollution, hyperlocal air pollution monitoring is necessary, but the existing regulatory monitoring networks are typically sparse due to the high costs to cover a full city area at the necessary spatial granularity. In this paper, we use the city of Antwerp in Belgium as a pilot to analyze the temporal and spatial distribution of four atmospheric pollutants $\left(\mathrm{NO}_{2}, \mathrm{PM}_{1}, \mathrm{PM}_{2.5}\right.$ and $\left.\mathrm{PM}_{10}\right)$ at street level by using mobile air pollution monitoring. In particular, we explore how the atmospheric pollutant concentration is affected by different context factors (e.g., road type, land use, source proximity). Our results demonstrate that these factors have an impact on the concentration distribution of the considered pollutants. For example, higher atmospheric $\mathrm{NO}_{2}$ concentrations are observed on primary roads, compared to secondary roads, and some source locations such as traffic lights have shown to be hot spots of atmospheric $\mathrm{NO}_{2}$ accumulation. These findings can be useful in order to formulate future local air quality measures and further improve current air quality models based on the observed impact of the considered context factors.
\end{abstract}

Keywords: Air pollution monitoring · Smart city · Internet of Things

\section{Introduction}

With industrial prosperity, urban development and growing traffic, the air pollution caused by the combustion of fossil fuels such as coal, oil and natural gas has gradually attracted worldwide attention due to its great threat to human 
health and to the natural environment. On one hand, atmospheric pollutants have a significant negative impact on human health, leading to cardiovascular diseases, lung cancer and thus reducing life expectancy. On the other hand, air pollution is responsible for many environmental problems, such as eutrophication and acidification of ecosystems.

The European Environment Agency (EEA) has listed seven types of atmospheric pollutants that people may be exposed to: particulate matter (PM), ozone $\left(\mathrm{O}_{3}\right)$, nitrogen dioxide $\left(\mathrm{NO}_{2}\right)$, sulfur dioxide $\left(\mathrm{SO}_{2}\right)$, carbon monoxide (CO), heavy metals, as well as benzene and benzopyrene [3]. In Europe, the most problematic pollutants in terms of harm to human health are particulate matter $(\mathrm{PM})$, nitrogen dioxide $\left(\mathrm{NO}_{2}\right)$ and ground-level ozone $\left(\mathrm{O}_{3}\right)$ [1]. In an urban environment, particularly at street level, these pollutants are directly or indirectly related to the process of burning fossil fuels such as road transport, electricity generation, industry and households [2].

Unfortunately, many European citizens live in places with serious air pollution. Air pollution monitoring is imperative to provide the government accurate data to assess air quality and the influence of counter measures such as low emission zones. It also provides the public with detailed and accurate air pollution information. This can help them plan some activities (e.g., location and time of sports activities). Nowadays, air pollution is measured by regulatory networks of static monitoring stations. Tian et al. [20] investigated the relationships between air pollution and various factors in the urban landscape including socioeconomic, urban form, and morphological characteristics based on hourly data at 35 monitoring stations in Beijing.

Although the static measuring stations are highly reliable and able to accurately measure various pollutants, the network of these stations is not suitable for street-level air pollution monitoring since the pollutants, especially trafficrelated, can show high spatial and temporal variability within a small neighborhood. Their spatial resolution is typically sparse due to the high installation and maintenance costs $(1 \sim 10 \mathrm{~km}$ [11]). For example, there are currently only 108 static measuring stations in Belgium, a country with an area of 30,688 square kilometers and a population of more than 11.4 million inhabitants [12]. Therefore, the static measuring stations may not always accurately characterize the high spatio-temporal variation in atmospheric pollutant concentration at street level and may thus not be representative for the whole city.

Meanwhile, advances in sensor technology and the emergence of portable and lower-cost sensing devices give rise to new opportunities for mobile air pollution monitoring. There have been many studies on the feasibility of mobile monitoring to measure air pollution at the high spatial and temporal resolution [23]. SM et al. [19] developed a smart personal air quality monitoring system (SPAMS) for urban air quality monitoring and personal exposure assessment. The monitoring campaign was designed to assess both pedestrian and public transport passenger exposure in Chennai city, India. The pedestrian exposure monitoring was carried out at three locations for 10 days, whereas personal exposure monitoring while travelling in bus was carried out at selected routes over a period of three months. 
In order to achieve fine-grained and realtime air pollution monitoring, Kaivonen et al. [10] deploy wireless sensors on public buses running on two selected routes to complement the coverage of stationary sensors in the city center of Uppsala, Sweden. McKercher et al. [14] assess the capability of low-cost mobile monitors to acquire useful data in a city without a monitoring network in place based on 30 days of data resulted from a bicycle platform along a $13.4 \mathrm{~km}$ fixed concentric route in Lubbock, Texas. Van den Bossch et al. [5] explored the potential of opportunistic mobile monitoring to map exposure to air pollution in an urban environment at a high spatial resolution. This was based on a total of 393 hours of measurements collected by city wardens in Antwerp, Belgium. Hofman et al. [8] evaluated personal exposure to ultrafine particle (UFP), black carbon (BC) and heavy metals while cycling near Antwerp, Belgium. The mobile monitoring was performed along two commuting routes for about two months.

However, these studies are often limited in spatial and/or temporal coverage. This is because they usually choose certain locations and time periods to carry out the monitoring campaigns. For example, the studies in [10] and [14] choose to use fixed routes to move carries (buses and bicycles). The fixed and short routes obviously can not prove the impact of various context factors on atmospheric pollutant concentration. Besides, the duration of monitoring campaigns in [19], [14] and [5] is very short, ranging from ten days to several months, which ignores the effects of climate and seasonality on atmospheric pollutant concentration. In our study, we perform a one-year opportunistic mobile monitoring campaign, where the routes of mobile sensors cover almost the entire city center of Antwerp.

The main contribution of this paper is in the following aspects: (1) we provide a systematic guideline on how to process and analyze air pollution datasets with time sequence and geographic information; (2) we analyze the temporal and spatial distribution of the considered pollutants and investigate the impact of various context factors (e.g., road type, land use and different emission sources) on the atmospheric pollutant concentration, which will facilitate the construction of a new air quality model [7] [6] in the future.

The rest of the paper is organized as follows. Section 2 introduces the methods and dataset adopted in this study. Section 3 describes and discusses some results we have found in present study and section 4 presents the conclusions.

\section{Material and Methods}

This section firstly describes the monitoring campaign that this study used to collect air pollution data and the details of the collected dataset. Secondly, this section provides a systematic guideline on how to process and analyze air pollution datasets with time sequence and geographic information, as shown in Fig. 1.

\subsection{Data Collection}

Opportunistic mobile monitoring is defined by [5] as a data collection method that installs the measurement devices on existing mobile sensor platforms. It differs from targeted mobile monitoring in that the measurement devices in targeted 


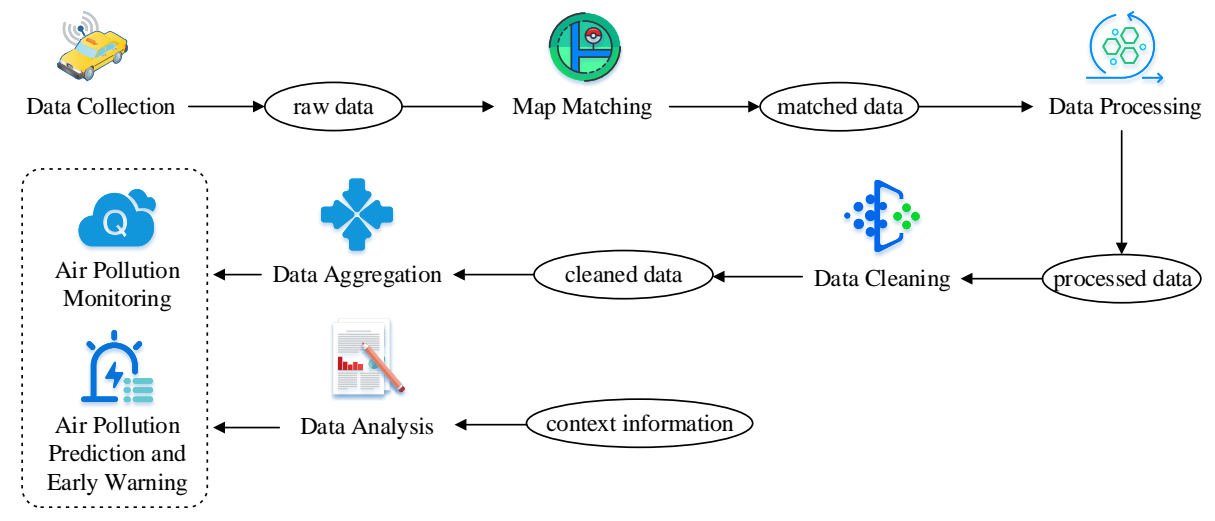

Fig. 1. The main workflow of the system

mobile monitoring follow the fixed routes designed by the researcher in advance, while the measurement devices in opportunistic mobile monitoring follow the daily routines of existing mobile sensor platforms. Therefore, opportunistic mobile monitoring enables the collection of large amounts of data at a relatively small cost.

The data used in our study has been collected as part of the opportunistic mobile monitoring campaign in Antwerp (with a population of 520,504 inhabitants, covering an area of $204.5 \mathrm{~km}^{2}$ ), Belgium based on the City of Things (CoT) framework [18]. 20 air quality sensors were mounted on the roofs of the Bpost (Belgian Post Group, the Belgian company responsible for the delivery of national and international mail) vans since January 2018. In this paper, we use the data from January to December 2018. These sensors deliver a record every 30 seconds, including measurements of four pollutants $\left(\mathrm{NO}_{2}\right.$ and three particulate matters with aerodynamic diameters below $1 \mu m\left(\mathrm{PM}_{1}\right), 2.5 \mu m\left(\mathrm{PM}_{2.5}\right)$ and 10 $\left.\mu m\left(\mathrm{PM}_{10}\right)\right)$ and meteorological information, such as temperature and relative humidity, which are linked with corresponding GPS locations and time stamps. As each Bpost car is driving around in the city, the set of sensors can cover the entire city in terms of measurements enabling the collection of real-time air quality information with broad city coverage, as opposed to an approach with static sensors, which only allows for local information. Furthermore, the number of static sensors necessary to cover the entire city is huge when compared to the needed number of cars and, thus, the installation and maintenance costs are also higher, which represent a considerable restriction when extending these kind of deployments.

\subsection{Map Matching}

In an urban environment, the occlusion of buildings will affect the reception of GPS signals. Coupled with the geographical error of the GPS device itself, we 
found that there is occasionally a big difference between the GPS location of the collected data points and their actual location.

The simplest way to improve the quality of the collected data points is to snap their locations to the nearest road segments. However, this method has great drawbacks. Without considering the consistency of the trajectories, it may lead to a point being snapped to an inconsistent road, or successive data points jumping from one road to another. Specifically, this can result in unfeasible trajectories, e.g., with unconnected roads.

To overcome this issue, Newson et al. [16] present an approach to use a Hidden Markov Model (HMM) to select the best candidate by combining the spatial and temporal component. Therefore, unconnected roads cannot be candidates and trajectories of data points must be consistent with the road network. For each GPS location, a number of map matching candidates within a certain radius around the GPS point is computed. To complement this approach, Luxen et al. [13] exploit the Viterbi algorithm to compute the most likely sequence of map matching candidates. An implementation of this approach is available in OSRM (Open Source Routing Machine) project. This framework can use a referenced road network (e.g., OpenStreetMap) to generate a hierarchical routing network. Xie et al. [22] proposed a novel approach to infer the road network by aligning the tracks for each road segment using a stretching and compression strategy. In this paper, we applied OSRM project to improve the data quality. Fig. 2 displays an example of the original locations and the improved locations after map-matching.

\subsection{Data Processing and Data Cleaning}

Further processing steps include filtering out data located outside of the study area and data not in working hours. These are described in more detail later in this section.

Step 1. filtering out data outside the study area This study focuses on the city of Antwerp. To determine which measurements are located in the study area, we set two simple thresholds: $51.1430<$ latitude $<51.3780$ and $4.2170<$ longitude $<4.4980$. We found that more than $90 \%$ of the measurements for each pollutant were located in the city of Antwerp.

Step 2. activity pattern detection and filtering out off-hour data Since the mobile sensors are measuring continuously day and night, we need to determine the working period to distinguish when the vehicle is on the road (working hours) and when it is parked in the garage (off-hours). We calculated the vehicle speed $(\mathrm{km} / \mathrm{h})$ from the geographical locations of subsequent measurements. Then, we applied the vehicle speed to detect the daily activity pattern of these devices.

As shown in Fig. 3, the daily working periods (working hours) can be defined as follows: 
X. Qin et al.

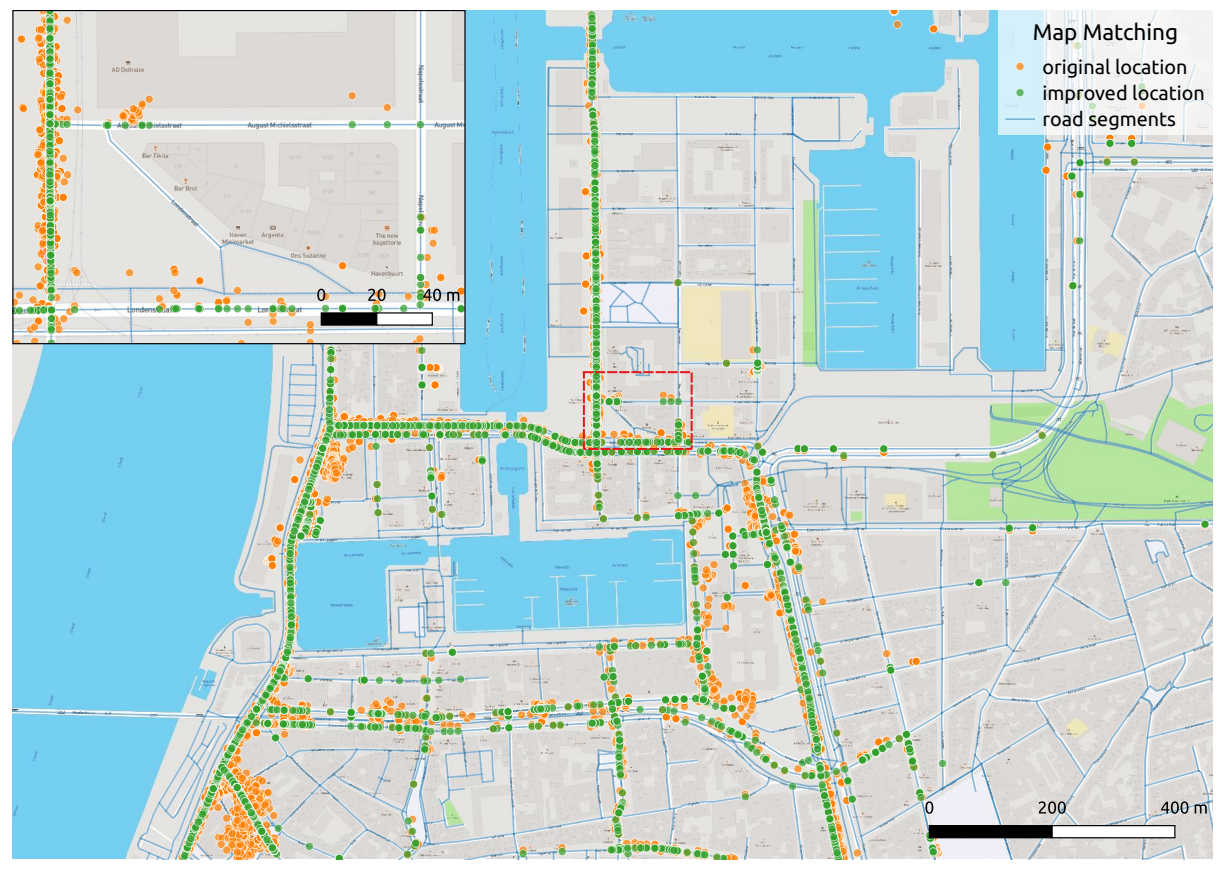

Fig. 2. Spatial data quality improvement by map matching

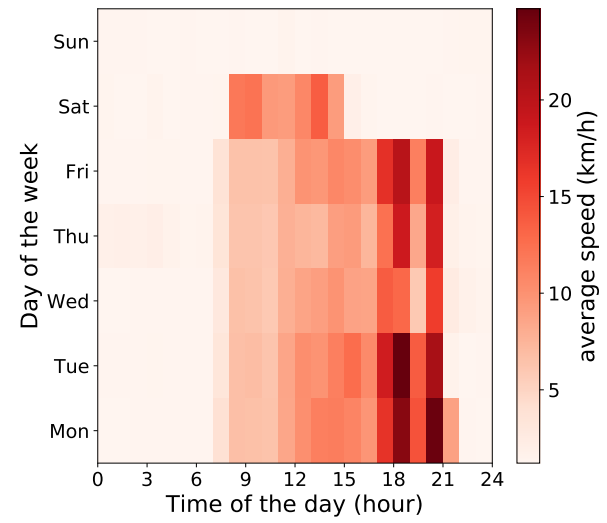

Fig. 3. The activity pattern of vehicles. It indicates the average speed $(\mathrm{km} / \mathrm{h})$ of vehicles for each hour and each day of the week. 
- Monday to Friday: 08:00 21:00

- Saturday: 08:00 15:00

- Sunday: no activity

Based on that, nearly $40 \%$ of the collected data is recorded in the streets, and is used in our analysis.

In this study, we measured, processed and analyzed four pollutants $\mathrm{NO}_{2}$, $\mathrm{PM}_{1}, \mathrm{PM}_{2.5}$ and $\mathrm{PM}_{10}$. In the following sections, we only choose one or two pollutants (e.g., $\mathrm{NO}_{2}$ and $\mathrm{PM}_{2.5}$ ) to show representative analysis results, since our main purpose is to use Antwerp as a pilot to illustrate the feasibility of the system, rather than investigating the actual urban pollution levels.

\subsection{Data Aggregation}

Spatial Variability We generate the air pollution map at street level for the entire city to see if we can identify general distribution patterns of these pollutants. Fig. 4 shows the resulting map of atmospheric $\mathrm{NO}_{2}$ concentration. Different colors represent different $\mathrm{NO}_{2}$ concentration levels. The darker the color, the higher the $\mathrm{NO}_{2}$ concentration level and the worse the air quality. It is obvious that the concentration of $\mathrm{NO}_{2}$ on the main roads is much higher compared to secondary roads in residential areas. We can also find that the concentration of these pollutants exhibits high spatial variability in the considered urban environment. The concentration of $\mathrm{NO}_{2}$ in two adjacent streets may vary considerably.

Temporal Variability All analyses of the temporal variation in atmospheric concentrations in this paper are based on the work-hour data, as defined earlier. From Fig. 5, we can observe the daily and weekly distribution patterns of the exhibited $\mathrm{NO}_{2}$ and $\mathrm{PM}_{2.5}$ concentrations. During the day, atmospheric concentrations of $\mathrm{NO}_{2}$ and $\mathrm{PM}_{2.5}$ increase during the morning and evening rush hours, especially during the morning peak (at 8 am and $9 \mathrm{am}$ ). This is probably because the morning rush hour is more concentrated between 9 am and $10 \mathrm{am}$, whereas the evening rush hour seems to be spread out over a longer period of time $(4 \mathrm{am} \sim 8 \mathrm{am})$. During the week, the concentrations of $\mathrm{NO}_{2}$ and $\mathrm{PM}_{2.5}$ in working days are generally higher than that on Saturdays. This pronounced diurnal variation, including rush hour peaks and difference between working and weekend days is typically observed for traffic-related pollution in urban environments [8] [9] [21] [17] [15].

\section{Result and Discussion}

In this section, a series of data analysis tasks are conducted to understand the relationship between the considered pollutants and various context factors (e.g., road type, land use) and then find whether these context factors have an effect on the concentration of pollutants. 
X. Qin et al.

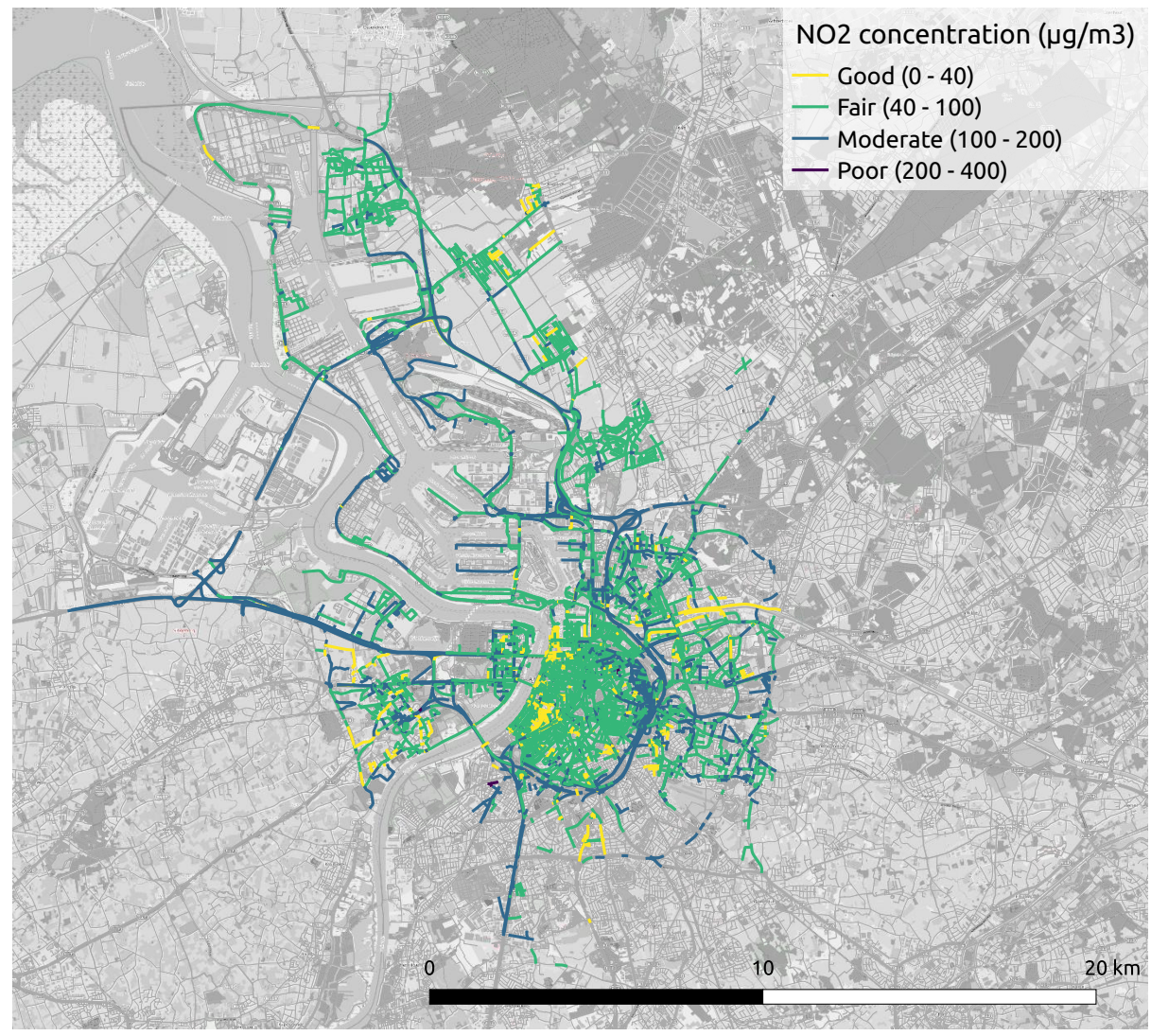

Fig. 4. Map of $\mathrm{NO}_{2}$ concentration: average $\mathrm{NO}_{2}$ concentration for each road segment with at least one measurement. The $\mathrm{NO}_{2}$ concentration levels correspond to the European Air Quality Index [4]. 


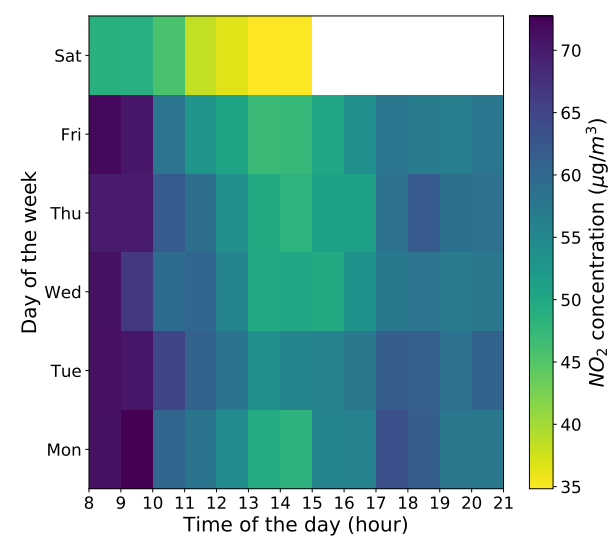

(a)



(b)

Fig. 5. The temporal pattern of (a) $\mathrm{NO}_{2}$ and (b) $\mathrm{PM}_{2.5}$. It indicates the average $\mathrm{NO}_{2}$ and $\mathrm{PM}_{2.5}$ concentrations $\left(\mu \mathrm{g} / \mathrm{m}^{3}\right)$ for each work-hour of the day and each day of the week.

\subsection{Road Type: Primary Roads and Secondary Roads}

In this study, the primary roads refer to roads with reference number like N173 and E19, as shown in Fig. 6. In fact, these primary roads usually have high traffic flow as they connect popular regions and have higher capacity. As for secondary roads, they tend to have relatively lower traffic capacity but higher traffic density. In this paper, traffic flow refers to the number of vehicles passing a reference point per unit of time while traffic density is defined as the number of vehicles per unit length of the road. From Fig. 7(a), we can see that the $\mathrm{NO}_{2}$ concentration on primary roads is slightly shifted towards higher values. Based on the T-test, there is a statistically significant difference in the $\mathrm{NO}_{2}$ concentrations observed along primary roads and secondary roads $(p<0.05)$. Also, the $\mathrm{NO}_{2}$ concentration on primary roads is always higher than that on secondary roads during most hours of the day, as shown in Fig. 7(b). This confirms that the road type has a significant impact on the distribution of $\mathrm{NO}_{2}$ concentration. The ring road is a 6-8 lane road with a much higher capacity, when compared to a singlelane secondary road. This higher capacity (vehicles per hour per lane) will lead to the higher atmospheric $\mathrm{NO}_{2}$ concentrations.

\subsection{Source Proximity: Traffic Signals}

The reason that we are interested in the locations of traffic signals is that these locations usually have relatively high traffic density and are prone to congestion, as the vehicles stop at the traffic signals frequently and many traffic signals are deployed at road intersections. We define a threshold to determine if a measurement is close to the traffic signals. In this study, we set the threshold to 30 meters. If the distance between a point and a traffic signal is less than 30 meters, 


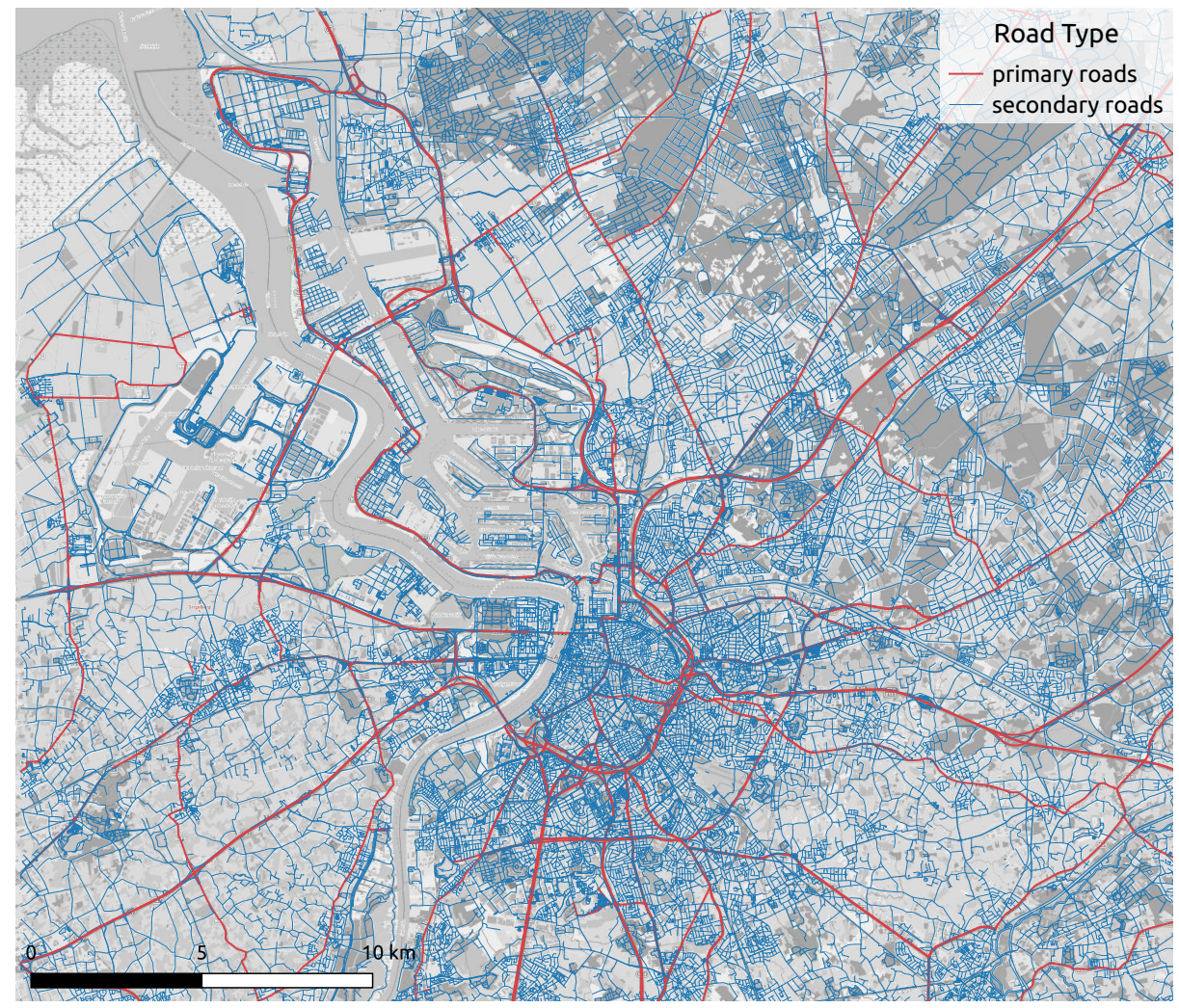

Fig. 6. Classification of road types in Antwerp, where the red lines represent the primary roads and the blue lines represent the secondary roads. 


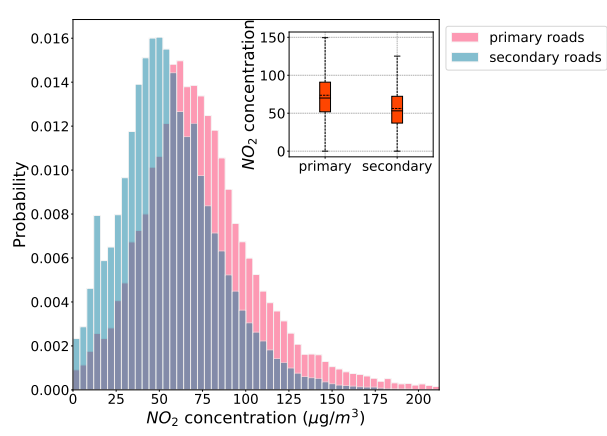

(a)

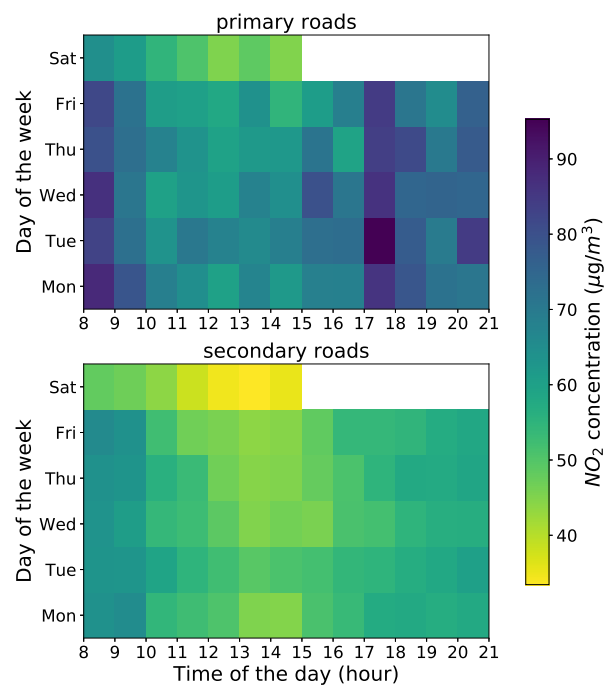

(b)

Fig. 7. (a) The normalized histogram and boxplot of the $\mathrm{NO}_{2}$ concentration on primary roads and secondary roads: they both indicate the $\mathrm{NO}_{2}$ concentration on primary roads is slightly shifted towards higher values; (b) The temporal distribution of $\mathrm{NO}_{2}$ concentration on primary roads and secondary roads: it indicates the average $\mathrm{NO}_{2}$ concentration on the two kinds of roads for each hour of the day and each day of the week. 
then the point belongs to the subset close to the traffic signals, otherwise the point belongs to the other subset. From Fig. 8(a), we can find that the $\mathrm{NO}_{2}$ concentration close to the traffic signals is slightly shifted towards higher values. As confirmed by the T-tests result $(p<0.05)$, there is a clear difference in the $\mathrm{NO}_{2}$ concentration close to traffic signals and others. Fig. 8(b) shows the $\mathrm{NO}_{2}$ concentration of data close to traffic signals is higher during most time of the day and shows higher temporal variability within a day.

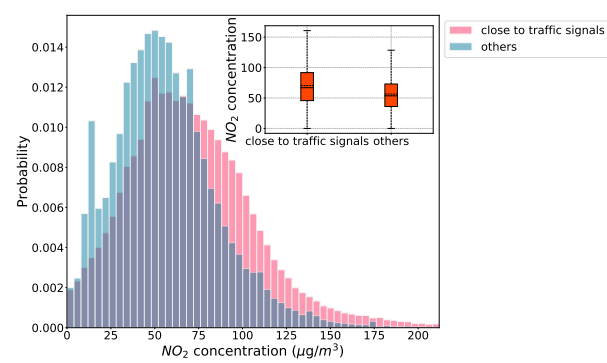

(a)

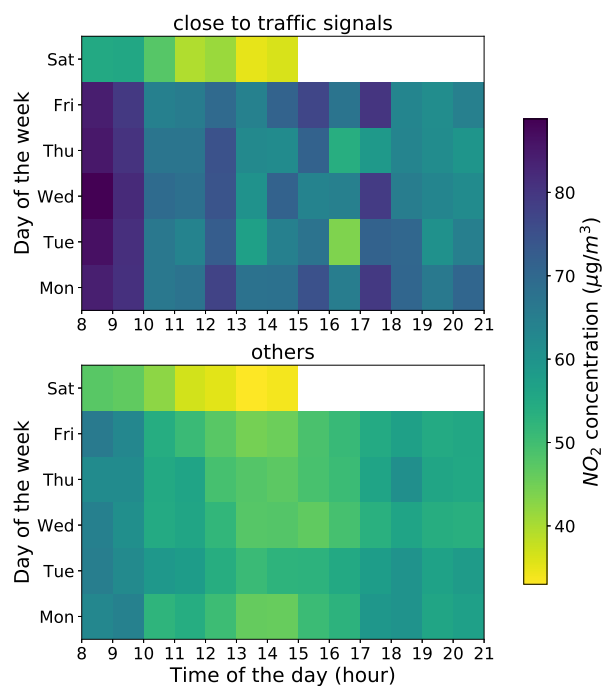

(b)

Fig. 8. (a) The normalized histogram and boxplot of the $\mathrm{NO}_{2}$ concentration close to traffic signals and others: they both indicate the $\mathrm{NO}_{2}$ concentration close to traffic signals and others is slightly shifted towards higher values; (b) The temporal distribution of $\mathrm{NO}_{2}$ concentration close to traffic signals and others: it indicates the average $\mathrm{NO}_{2}$ concentration of these two subsets for each hour of the day and each day of the week.

\subsection{Land Use: Residential Regions and Industrial Regions}

In this study, we acquire the land use types from OpenStreetMap, a collaborative project to create a free editable map of the world, as shown in Fig. 9. We define a threshold to decide whether a point belongs to the industrial region or residential region. In this study, we set the threshold to 10 meters. If the distance from a point to the industrial/residential region is less than 10 meters, then this point belongs to the industrial/residential region.

From Fig. 10, for both $\mathrm{NO}_{2}$ and $\mathrm{PM}_{2.5}$, we can observe that the concentration in residential regions is slightly shifted towards higher values compared to that in industrial regions. According to T-tests result $(p<0.05)$, there is a 


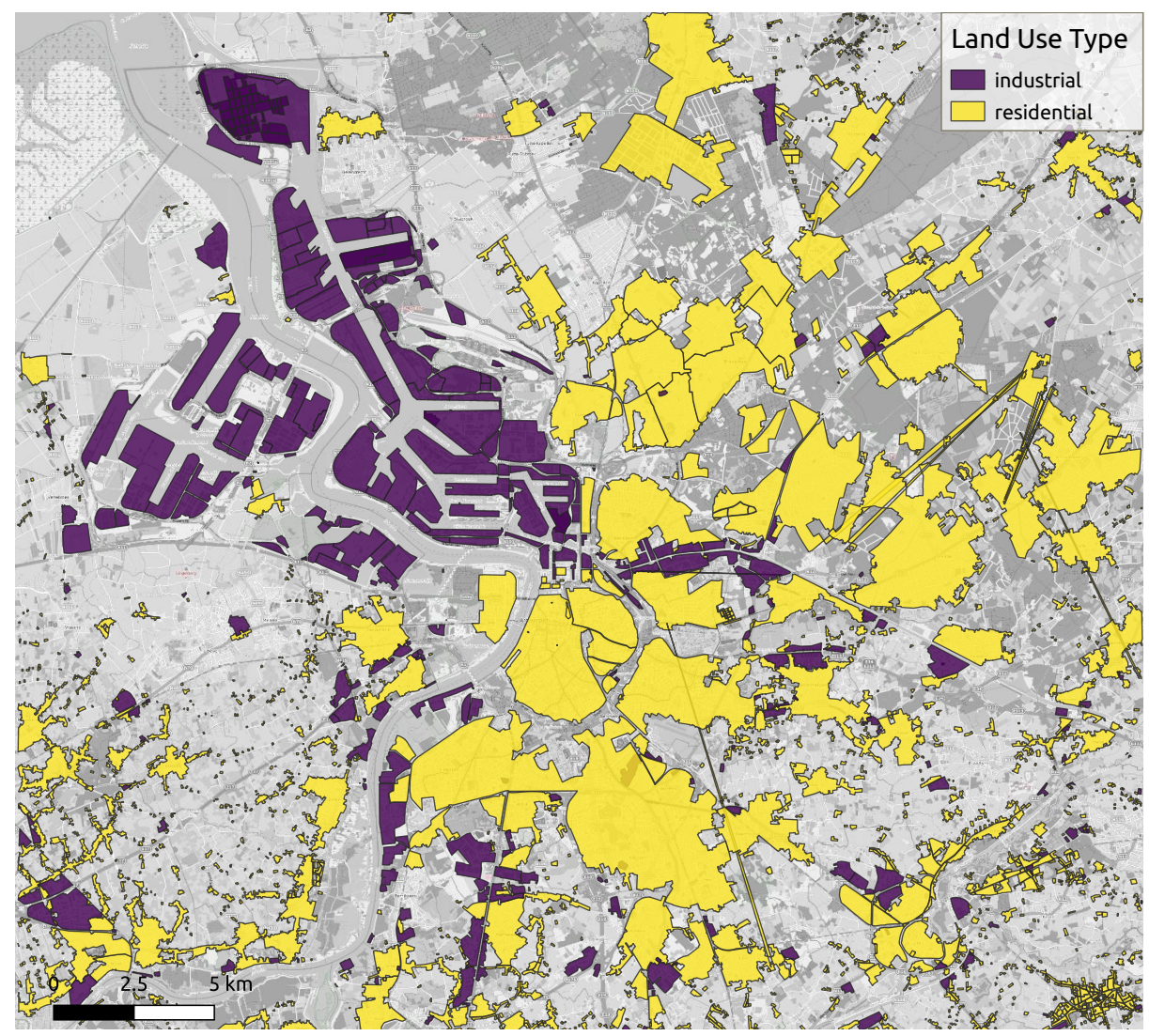

Fig. 9. Classification of land use types in Antwerp, where the purple polygons represent the industrial area and the yellow polygons represent the residential area. 


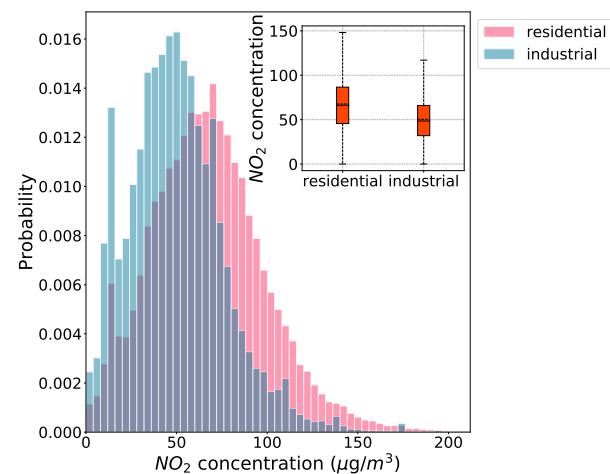

(a)

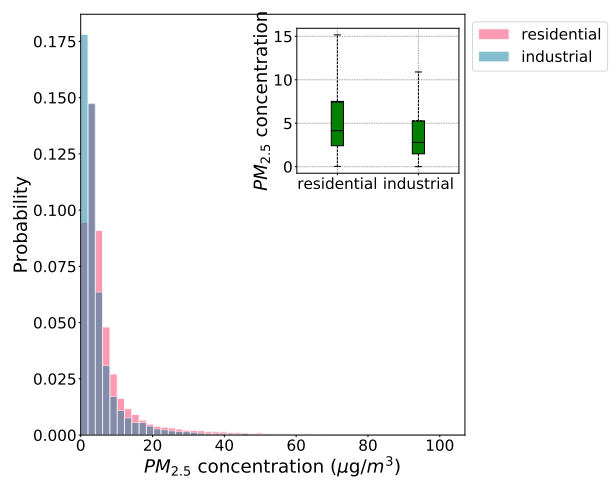

(b)

Fig. 10. The normalized histograms and boxplots of the (a) $\mathrm{NO}_{2}$ and (b) $\mathrm{PM}_{2.5}$ concentrations in industrial regions and residential regions: they indicate for both $\mathrm{NO}_{2}$ and $\mathrm{PM}_{2.5}$, the concentrations in residential regions are higher than that in industrial regions.

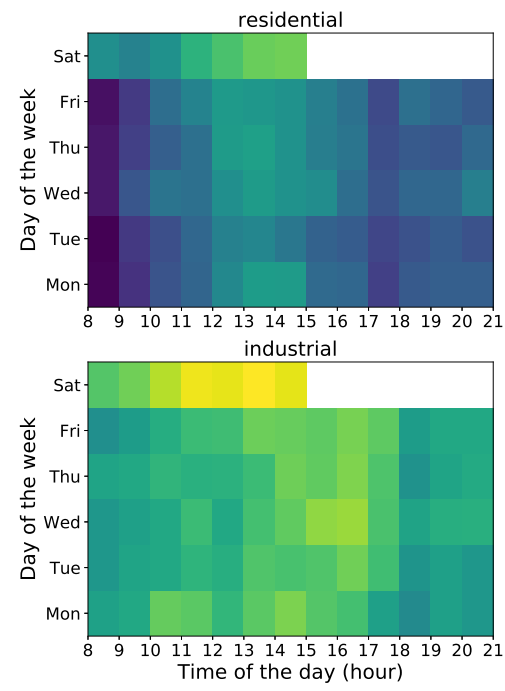

(a)

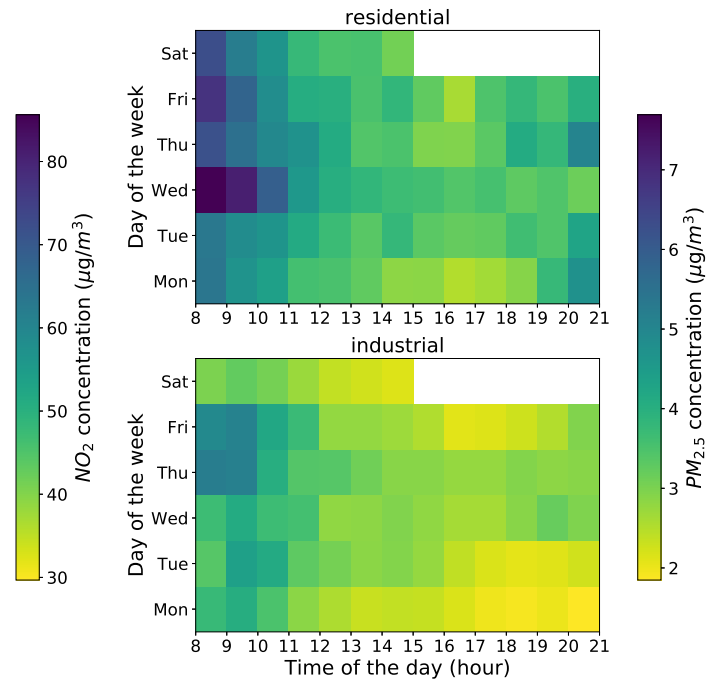

(b)

Fig. 11. The temporal distribution of (a) $\mathrm{NO}_{2}$ and (b) $\mathrm{PM}_{2.5}$ concentrations in industrial regions and residential regions: it indicates the average $\mathrm{NO}_{2}$ and $\mathrm{PM}_{2.5}$ concentrations in these two kinds of regions for each hour of the day and each day of the week. 
significant difference between the concentrations in industrial regions and residential regions. Fig. 11 shows the $\mathrm{NO}_{2}$ and $\mathrm{PM}_{2.5}$ concentrations in residential regions are always higher than that in industrial regions during most time of the day. This is not surprising as this study focuses on urban traffic-related pollutants (e.g., $\mathrm{NO}_{2}$ and $\mathrm{PM}$ ), while industry in the city of Antwerp is mainly petrochemical. Due to the obvious higher traffic density in the city center and urban architecture impeding natural ventilation, higher pollutant concentrations can be expected in the residential areas.

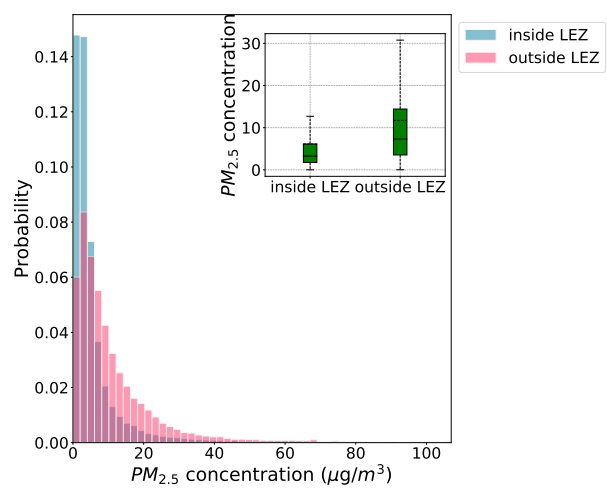

(a)



(b)

Fig. 12. (a) The normalized histogram and boxplot of the $\mathrm{PM}_{2.5}$ concentration inside LEZ and outside LEZ: they both indicate the $\mathrm{PM}_{2.5}$ concentration outside LEZ is slightly shifted towards higher values; (b) The temporal distribution of $\mathrm{PM}_{2.5}$ concentration inside LEZ and outside LEZ: it indicates the average $\mathrm{PM}_{2.5}$ concentration of these two subsets for each hour of the day and each day of the week.

\subsection{Low Emission Zone}

Since February 2017, the city of Antwerp has introduced a Low Emission Zone (LEZ) in the entire city center. The entry restrictions of the LEZ will be gradually tightened. In the first stage between 2017 and 2020, vehicles with high pollutant levels are no longer permitted to enter the environmental zone. The LEZ of Antwerp is permanent, that means 24 hours a day for 7 days a week, also on Sundays and public holidays. The environmental zone covers $20 \mathrm{~km}^{2}$ and affects about 200,000 inhabitants. Fig. 12(a) shows that the PM2.5 concentration outside LEZ is hugely shifted towards higher values. In addition to the T-tests 
result $(p<0.05)$, there is a significant difference in the $\mathrm{PM}_{2.5}$ concentration inside LEZ and outside LEZ. As shown in Fig. 12(b), $\mathrm{PM}_{2.5}$ concentration outside LEZ is always higher than that inside LEZ during most time of the day. This may be because in the face of additional taxes, people are more likely to choose environmentally friendly modes of travel, such as taking a bus or riding a bicycle instead of taking a private car with high emissions.

\section{Conclusion and Further Steps}

First of all, this study proofs the feasibility of collecting meaningful air quality data from opportunistic mobile monitoring platforms. Based on the data collected from Antwerp, we analyzed the spatial and temporal distribution of the considered pollutants $\left(\mathrm{NO}_{2}\right.$ and $\left.\mathrm{PM}\right)$ and found that the atmospheric pollutant concentration is highly variable in both time and space. The results show the value of fine-grained air pollution monitoring. We also identified general distribution patterns of the considered pollutants. For example, the main roads are very conspicuous in the $\mathrm{NO}_{2}$ concentration map. In particular, we investigated the impact of various context factors (e.g., road type, land use and some emission sources) on the atmospheric pollutant concentration. For example, some source locations, such as traffic signals, tend to have higher $\mathrm{NO}_{2}$ concentration levels. We believe that these findings are of great value in assessing current air pollution control measures and formulating future air quality improvement measures.

Next, we intend to construct more effective air quality prediction and air pollution early warning models based on the context aware analysis.

Acknowledgments. This research was supported by the Internet of Things (IoT) team of imec-Netherlands under the project EI2.

\section{References}

1. Agency, E.E.: Air pollution. https://www.eea.europa.eu/themes/air/intro, accessed April 24, 2019

2. Agency, E.E.: Air pollution sources. https://www.eea.europa.eu/themes/air/ air-pollution-sources, accessed April 24, 2019

3. Agency, E.E.: Air quality in europe 2011 report. https://www.eea.europa.eu/ publications/air-quality-in-europe-2011, accessed April 24, 2019

4. Agency, E.E.: European air quality index. http://airindex.eea.europa.eu/, accessed April 24, 2019

5. Van den Bossche, J., Theunis, J., Elen, B., Peters, J., Botteldooren, D., De Baets, B.: Opportunistic mobile air pollution monitoring: a case study with city wardens in antwerp. Atmospheric environment 141, 408-421 (2016)

6. Deligiannis, N., Mota, J.F., Zimos, E., Rodrigues, M.R.: Heterogeneous networked data recovery from compressive measurements using a copula prior. IEEE Transactions on Communications 65(12), 5333-5347 (2017) 
7. Do, T.H., Nguyen, D.M., Tsiligianni, E., Aguirre, A.L., La Manna, V.P., Pasveer, F., Philips, W., Deligiannis, N.: Matrix completion with variational graph autoencoders: Application in hyperlocal air quality inference. In: ICASSP 2019-2019 IEEE International Conference on Acoustics, Speech and Signal Processing (ICASSP). pp. 7535-7539. IEEE (2019)

8. Hofman, J., Staelens, J., Cordell, R., Stroobants, C., Zikova, N., Hama, S., Wyche, K., Kos, G., Van Der Zee, S., Smallbone, K., et al.: Ultrafine particles in four european urban environments: Results from a new continuous long-term monitoring network. Atmospheric environment 136, 68-81 (2016)

9. de Jesus, A.L., Rahman, M.M., Mazaheri, M., Thompson, H., Knibbs, L.D., Jeong, C., Evans, G., Nei, W., Ding, A., Qiao, L., et al.: Ultrafine particles and pm2. 5 in the air of cities around the world: Are they representative of each other? Environment international 129, 118-135 (2019)

10. Kaivonen, S., Ngai, E.: Real-time air pollution monitoring with sensors on city bus. Digital Communications and Networks (2019)

11. Karner, A.A., Eisinger, D.S., Niemeier, D.A.: Near-roadway air quality: synthesizing the findings from real-world data. Environmental science \& technology 44(14), $5334-5344(2010)$

12. voor het Leefmilieu (IRCEL), I.C.: Measurements - english. http://www . irceline.be/en/air-quality/measurements/measurements?set_language=en, accessed April 24, 2019

13. Luxen, D., Vetter, C.: Real-time routing with openstreetmap data. In: Proceedings of the 19th ACM SIGSPATIAL international conference on advances in geographic information systems. pp. 513-516. ACM (2011)

14. McKercher, G.R., Vanos, J.K.: Low-cost mobile air pollution monitoring in urban environments: a pilot study in lubbock, texas. Environmental technology 39(12), 1505-1514 (2018)

15. Mishra, V.K., Kumar, P., Van Poppel, M., Bleux, N., Frijns, E., Reggente, M., Berghmans, P., Panis, L.I., Samson, R.: Wintertime spatio-temporal variation of ultrafine particles in a belgian city. Science of the total environment 431, 307-313 (2012)

16. Newson, P., Krumm, J.: Hidden markov map matching through noise and sparseness. In: Proceedings of the 17th ACM SIGSPATIAL international conference on advances in geographic information systems. pp. 336-343. ACM (2009)

17. Pirjola, L., Lähde, T., Niemi, J., Kousa, A., Rönkkö, T., Karjalainen, P., Keskinen, J., Frey, A., Hillamo, R.: Spatial and temporal characterization of traffic emissions in urban microenvironments with a mobile laboratory. Atmospheric Environment 63, 156-167 (2012)

18. Santos, J., Vanhove, T., Sebrechts, M., Dupont, T., Kerckhove, W., Braem, B., Van Seghbroeck, G., Wauters, T., Leroux, P., Latre, S., et al.: City of things: Enabling resource provisioning in smart cities. IEEE Communications Magazine 56(7), 177-183 (2018)

19. SM, S.N., Yasa, P.R., Narayana, M., Khadirnaikar, S., Rani, P.: Mobile monitoring of air pollution using low cost sensors to visualize spatio-temporal variation of pollutants at urban hotspots. Sustainable Cities and Society 44, 520-535 (2019)

20. Tian, Y., Yao, X., Chen, L.: Analysis of spatial and seasonal distributions of air pollutants by incorporating urban morphological characteristics. Computers, Environment and Urban Systems 75, 35-48 (2019)

21. Wang, Y., Ying, Q., Hu, J., Zhang, H.: Spatial and temporal variations of six criteria air pollutants in 31 provincial capital cities in china during 2013-2014. Environment international 73, 413-422 (2014) 
22. Xie, X., Philips, W., Veelaert, P., Aghajan, H.: Road network inference from gps traces using dtw algorithm. In: 17th International IEEE Conference on Intelligent Transportation Systems (ITSC). pp. 906-911. IEEE (2014)

23. Xie, X., Semanjski, I., Gautama, S., Tsiligianni, E., Deligiannis, N., Rajan, R., Pasveer, F., Philips, W.: A review of urban air pollution monitoring and exposure assessment methods. ISPRS International Journal of Geo-Information 6(12), 389 (2017) 